\title{
EMBEDDING OF MODULES OVER GORENSTEIN RINGS
}

\author{
HANS-BJØRN FOXBY
}

\begin{abstract}
Let $A$ be a noetherian commutative ring which is a homomorphic image of a Gorenstein ring. The first result in this note is that $A$ is a Cohen-Macaulay ring if and only if every finitely generated $A$-module can be embedded in a finitely generated $A$ module $T$ which is pointwise of finite injective dimension (i.e. all localizations of $T$ are of finite injective dimension). The second result is that if $\boldsymbol{A}$ is Cohen-Macaulay, then every finitely generated $\boldsymbol{A}$ module can be embedded in a finitely generated $A$-module of finite projective dimension if and only if $A$ is Gorenstein.
\end{abstract}

Let $A$ denote a commutative noetherian ring. As a running hypothesis we will assume that $A$ is a homomorphic image of a Gorenstein ring (a commutative noetherian ring $R$ is Gorenstein if $R_{\mathfrak{p}}$ has finite injective dimension as an $R_{\mathfrak{p}}$-module, for every prime ideal $\mathfrak{p}$ of $R$; see [2] for other definitions equivalent to this one). However, this class of noetherian rings is rather extensive, since it contains most of the rings occurring in algebraic geometry, and, in particular, it contains the complete local rings, by Cohen's structure theorem [5].

All modules will be finitely generated (f.g.), and for a f.g. $A$-module $M$ and $\mathfrak{p} \in \operatorname{Spec} A$, depth $M_{\mathfrak{p}}$ denotes (as usual) the maximal length of a $M_{\mathfrak{p}^{-}}$ regular sequence in $\mathfrak{p} A_{\mathfrak{p}}$, while grade $M$ is the maximal length of an $A$-regular sequence in Ann $M$. Furthermore, recall that a f.g. nonzero $A$ module $\Omega$ is said to be a Gorenstein module if $\Omega$ pointwise is of finite injective dimension (i.e. inj $\operatorname{dim} \Omega_{\mathfrak{p}}<\infty$ for all $\mathfrak{p} \in \operatorname{Supp} \Omega$ ) and depth $\Omega_{\mathfrak{p}}=$ depth $A_{\mathfrak{p}}$ for all $\mathfrak{p} \in \operatorname{Supp} \Omega$. Since $A$ is a homomorphic image of a Gorenstein ring, by assumption, there exists a Gorenstein $A$-module which is of rank one (i.e. $\operatorname{Hom}(\Omega, \Omega)=A$ ), if $A$ is a Cohen-Macaulay ring (cf. Sharp [11], and see [10], [6] and [7] for further properties of Gorenstein modules).

Theorem 1. A is a Cohen-Macaulay ring if and only if every f.g. Amodule can be embedded in a f.g. A-module which pointwise is of finite injective dimension.

Received by the editors March 24, 1972.

AMS (MOS) subject classifications (1970). Primary 13H10; Secondary 13D05, $13 \mathrm{C} 15$. Key words and phrases. Cohen-Macaulay rings, Gorenstein rings, G-dimension. 
Proof OF "IF". The ring $A$ itself is a submodule of a f.g. module $T$ pointwise of finite injective dimension. For any prime ideal $\mathfrak{p}$ we have grade $_{A_{\mathfrak{p}}} T_{\mathrm{p}}=0$ and hence $A_{\mathfrak{p}}$ is a Cohen-Macaulay ring, by [9, Theorem 1.6].

Proof of "ONLY IF". Assume that $A$ is Cohen-Macaulay, let $\Omega$ be a Gorenstein module of rank one, and let $M$ be f.g. Let us assume $\{$ ht $\mathfrak{p} \mid \mathfrak{p} \in$ Ass $M\}=\left\{g_{1}, \cdots, g_{p}\right\}$ (i.e. $g_{1}, \cdots, g_{p}$ are the possible heights of a prime ideal in Ass $M$ ), and let $M_{i}$ be the intersection of those $\mathfrak{p}$ primary components of 0 in $M$ which have ht $\mathfrak{p}=g_{i}$, i.e. Ass $M / M_{i}=$ $\left\{\mathfrak{p} \in\right.$ Ass $M \mid$ ht $\left.\mathfrak{p}=g_{i}\right\}$ and $M_{1} \cap \cdots \cap M_{p}=0$, and hence we have a monomorphism $M \rightarrow M / M_{1} \oplus \cdots \oplus M / M_{p}$. We may therefore assume ht $p=g$ for all $p \in$ Ass $M$. We divide now in two cases:

$1^{\circ}$. $g=0$. Let $S$ denote the set of nonzero-divisors on $M$. Then $S$ is a multiplicative system in $A$, and the ring of fractions $S^{-1} A$ is artinian. $S^{-1} \Omega$ is a f.g. injective $S^{-1} A$-module, in fact, it is the direct sum of the $S^{-1} A$-injective hulls of the (finitely many) residue class fields of $S^{-1} A$, and hence the $S^{-1} A$-injective hull of $S^{-1} M$ can be embedded in the direct sum $\oplus m S^{-1} \Omega$ of $m$ copies of $S^{-1} \Omega$ (for a suitable number $m$ ). This shows that there exists a $S^{-1} A$-monomorphism $\varphi: S^{-1} M \rightarrow S^{-1} T$ where $T=\oplus m \Omega$. Now choose $s \in S$ and an $A$-homomorphism $\psi: M \rightarrow T$ such that $\psi(m) / s=$ $\varphi(m / 1)$ in $S^{-1} T$ for all $m \in M$ (cf. [4, $\$ 2$, No. 7, Proposition 19]). If $\psi(m)=0$ then $\varphi(m / 1)=0$ in $S^{-1} T$ and hence $m / 1=0$ in $S^{-1} M$, i.e. $m=0$ in $M$ (by the definition of $S$ ). This shows that $\psi$ is the desired monomorphism: $M \rightarrow T=\oplus m \Omega$.

$2^{\circ}$. $g>0$. Since $A$ is Cohen-Macaulay we have grade $M=$ ht Ann $M=$ $g>0$, and hence we may choose an $A$-regular sequence $a_{1}, \cdots, a_{g} \in$ Ann $M$ of length $g$. Now $\bar{A}=A /\left(a_{1}, \cdots, a_{g}\right)$ is a Cohen-Macaulay ring (and homomorphic image of a Gorenstein ring) and $\bar{\Omega}=\Omega \otimes \bar{A}$ is a Gorenstein $\bar{A}$-module of rank one. Furthermore, all the prime ideals in $\mathrm{Ann}_{\bar{A}} M$ are of $\bar{A}$-height zero, so, by case $1^{\circ}, M$ may be embedded in $\oplus m \bar{\Omega}$, which pointwise is of finite $A$-injective dimension.

REMARK. From the above proof it follows that if $A$ is Cohen-Macaulay and if $g_{1}, \cdots, g_{n}$ are the possible heights of ideals in Ass $M$, then there exist numbers $m_{1}, \cdots, m_{p}$ and $p A$-regular sequences $a_{i 1}, \cdots, a_{i g_{i}}$, $1 \leqq i \leqq p$, such that $M$ may be embedded in

$$
T=\oplus_{i=1}^{n}\left(\oplus m_{i} \Omega /\left(a_{i 1}, \cdots, a_{i g_{i}}\right) \Omega\right) .
$$

Here we have grade $T=$ grade $M$, and, if $A$ is local, $\operatorname{dim} T=\operatorname{dim} M$ and depth $T=\operatorname{depth} A-\sup g_{i}=\inf \{\operatorname{dim} A / \mathfrak{p} \mid \mathfrak{p} \in$ Ass $M\} \geqq \operatorname{depth} M$, see [2, 3.1]. 
Corollary. If $A$ is a Cohen-Macaulay ring and $M$ is a f.g. A-module which is pointwise of finite $G$-dimension, then $M$ can be embedded in a f.g.P with proj $\operatorname{dim} P=\sup \{$ ht $\mathfrak{p} \mid \mathfrak{p} \in$ Ass $M\} \leqq G-\operatorname{dim} M$ and grade $P=\operatorname{grade} M$. Here $P=\oplus_{i=1}^{p}\left(\oplus m_{i} A /\left(a_{i 1}, \cdots, a_{i p}\right)\right)$, where $m_{1}, \cdots, m_{p}$ are certain numbers, $\left\{g_{1}, \cdots, g_{p}\right\}=\{$ ht $\mathfrak{p} \mid \mathfrak{p} \in$ Ass $M\}$, and $a_{i 1}, \cdots, a_{i g_{i}}$ an A-regular sequence for all $i, 1 \leqq i \leqq p$.

(For definition of the concept " $G$-dimension" see [1, Chapter 3].)

Proof. Let again $\Omega$ denote a rank one Gorenstein module. For $N=$ $\Omega \otimes M$ we have $M=\operatorname{Hom}(\Omega, N)$, by [7, Proposition 2.4], since $M$ is pointwise of finite $G$-dimension. As in the above remark we have a monomorphism

$$
N \rightarrow T=\bigoplus_{i=1}^{p}\left(\oplus m_{i} \Omega \otimes A /\left(a_{i 1}, \cdots, a_{i g_{i}}\right)\right)
$$

and hence a monomorphism

since

$$
M \rightarrow \operatorname{Hom}(\Omega, T)=\bigoplus_{i=1}^{p}\left(\oplus m_{i} A /\left(a_{i 1}, \cdots, a_{i g_{i}}\right)\right),
$$

$$
\operatorname{Hom}\left(\Omega, \Omega \otimes A /\left(a_{i 1}, \cdots, a_{i g_{i}}\right)\right)=A /\left(a_{i 1}, \cdots, a_{i g_{i}}\right),
$$

by [7, Theorem 2.1] (or directly). Now the corollary is proved, since it is easy to prove that grade $N=$ grade $M$, Ass $N=$ Ass $M$, and, if $A$ is local, depth $N=\operatorname{depth} M$ (cf. [7, Proposition 2.4] and (the proof of) [6, Proposition 3.1, (b)]). Furthermore, we have (if $A$ is local)

$$
\begin{aligned}
\operatorname{proj} \operatorname{dim} P & =\sup g_{i}=\sup \{\text { ht } \mathfrak{p} \mid \mathfrak{p} \in \text { Ass } M\} \\
& \leqq \operatorname{depth} A-\operatorname{depth} M=G-\operatorname{dim} M
\end{aligned}
$$

(cf. [1, Theorem 4.13]).

It is well known that $A$ is a quasi-Frobenius ring (= an artinian Gorenstein ring) if and only if every f.g. $A$-module $M$ is a submodule of a f.g. free $A$-module (i.e. $M$ is torsionless), see [1, Theorem 2.6, (a) and (d)]. We are now able to prove the following extension of this:

THEOREM 2. The following three statements are equivalent:

(1) $A$ is a Gorenstein ring.

(2) $A$ is a Cohen-Macaulay ring and every f.g. A-module can be embedded in a f.g. A-module of finite projective dimension.

(3) Every f.g. A-module can be embedded in a f.g. A-module of same garde and of finite projective dimension.

Proof. $\quad(1) \Rightarrow(2)$ follows from the Corollary since every f.g. module over a Gorenstein ring pointwise is of finite $G$-dimension, cf. [1, Theorem 4.20] - or from Theorem 1 itself since every f.g. module pointwise of 
finite injective dimension over a Gorenstein ring is of finite projective dimension by [3, Chapter III, Corollary 6.6] and [8, Theorem 2.2].

$(2) \Rightarrow(3)$. Let us assume the following:

(2') $A$ is Cohen-Macaulay and every f.g. $M$ is a submodule of a f.g. $P$ which is pointwise of finite $G$-dimension.

It is trivial that $(2) \Rightarrow\left(2^{\prime}\right)$. Let $M$ be f.g., i.e. $M$ is a submodule of $P$, where $P$ is pointwise of finite $G$-dimension, and hence, by the corollary, $M$ is a submodule of $\oplus_{i=1}^{n} P_{i}$, where $P_{i}=\oplus m_{i} A /\left(a_{i 1}, \cdots, a_{i g_{i}}\right)$. Here we have grade $\oplus_{i=1}^{p} P_{i}=$ grade $P \leqq$ grade $M=g$. Assume $g_{i}<g$ for $i=1, \cdots, q$ and $g_{i} \geqq g$ for $i=q+1, \cdots, p(0 \leqq q \leqq p)$. For $x \in \oplus_{i=1}^{a} P_{i}-0$ we have ht Ann $x<$ $g$, since $A$ is Cohen-Macaulay, but for all $m \in M$ we have ht Ann $m \geqq g$, and hence $M$ is a submodule of $\oplus_{i=q+1}^{p} P_{i}$ which is of grade $\geqq g=\operatorname{grade} M$, and hence equal.

(3) $\Rightarrow(1)$. Assume that the following weak version of (3) holds:

$\left(3^{\prime}\right)$ Every f.g. $M$ is a submodule of a f.g. $P$ which is pointwise of finite $G$-dimension and with grade $P=$ grade $M$.

Let $\mathrm{m}$ be any maximal ideal in $A$ and let $a_{1}, \cdots, a_{d} \in \mathrm{m}$ be a maximal $A$-regular sequence, $d=$ grade $A / \mathrm{m}=\operatorname{depth} A_{\mathrm{m}}$ (cf. [1, 4.5]). Then the images of $a_{1}, \cdots, a_{d}$ in $A^{\prime}=A_{11}$ form a maximal $A^{\prime}$-regular sequence $a_{1}^{\prime}, \cdots, a_{d}^{\prime} \in \mathrm{m} A^{\prime}$, and we are going to prove that every f.g. module $M^{\prime}$ over $B=A^{\prime} /\left(a_{1}^{\prime}, \cdots, a_{d}^{\prime}\right)$ is a torsionless $B$-module. This will imply that $B$ is a quasi-Frobenius ring, and therefore that $A^{\prime}=A_{m}$ is a Gorenstein ring as desired.

Let $M^{\prime}$ be a f.g. $B$-module and choose a f.g. $A$-submodule $M$ of $M^{\prime}$ such that $M_{\mathrm{m}}=M^{\prime}$. Then $a_{1}, \cdots, a_{d}$ is an $A$-regular sequence in Ann $M$, and hence grade ${ }_{A} M=d$. By our assumption ( $\left.3^{\prime}\right), M$ can be embedded in a f.g. $P$ with grade $P=d$ and $G$ - $\operatorname{dim}_{A^{\prime}}, P^{\prime}<\infty$, where $P^{\prime}=P_{\mathrm{m}}$. Here we have $d \leqq \operatorname{grade}_{A} P \leqq \operatorname{grade}_{A^{\prime}} P^{\prime} \leqq \operatorname{depth} A^{\prime}=d$ (cf. [1, Theorem 4.13]). Now $P^{\prime}$ is of $G$-dimension zero over $\bar{A}=A^{\prime} /\left(x_{1}, \cdots, x_{d}\right)$ where $x_{1}, \cdots, x_{d}$ is an $A^{\prime}$-regular sequence in $\mathrm{Ann}_{A^{\prime}} P^{\prime} \subseteq \mathrm{Ann}_{A^{\prime}} M^{\prime}$, by [1, Proposition 4.35]. In particular, $P^{\prime}$ is a torsionless $\bar{A}$-module, and hence $M^{\prime}$ is a torsionless $\bar{A}$ module too, i.e. $M^{\prime}$ is a submodule of

$$
\begin{aligned}
\operatorname{Hom}_{\overline{.}}\left(\operatorname{Hom}_{\bar{A}}\left(M^{\prime}, \bar{A}\right), \bar{A}\right) & =\operatorname{Ext}_{A^{\prime}}^{d}\left(\operatorname{Ext}_{A^{\prime}}^{d}\left(M^{\prime}, A^{\prime}\right), A^{\prime}\right) \\
& =\operatorname{Hom}_{B}\left(\operatorname{Hom}_{B 3}\left(M^{\prime}, B\right), B\right)
\end{aligned}
$$

which is a torsionless $B$-module, and hence also $M$ is a torsionless $B$ module, and we are done.

\section{REFERENCES}

1. M. Auslander and M. Bridger, Stable module theory, Mem. Amer. Math. Soc. No. 94 (1969). MR 42 \#4580.

2. H. Bass, On the ubiquity of Gorenstein rings, Math. Z. 82 (1963), 8-28. MR 27 \# 3669. 
3. H. Bass, Algebraic K-theory, Benjamin, New York, 1968. MR 40 \#2736.

4. N. Bourbaki, Éléments de mathématique. Fasc. XXVII. Algèbre commutative. Chap. 2: Localisation, Actualités Sci. Indust., no. 1290, Hermann, Paris, 1961. MR 36 \#146.

5. I. S. Cohen, On the structure and ideal theory of complete local rings, Trans. Amer. Math. Soc. 59 (1946), 54-106. MR 7, 509.

6. H.-B. Foxby, On the $\mu^{i}$ in a minimal injective resolution, Math. Scand. 29 (1972), 175-186.

7. —_ Gorenstein modules and related modules, Math. Scand. (to appear).

8. G. Levin and W. V. Vasconcelos, Homological dimensions and Macaulay rings, Pacific J. Math. 25 (1968), 315-323. MR 37 \#6275.

9. C. Peskine and L. Szpiro, Modules de type et de dimension injective finie sur un anneau local noethérien, C. R. Acad. Sci. Paris Sér. A-B 266 (1968), A1117-A1120. MR 38 \#3266.

10. R. Y. Sharp, Gorenstein modules, Math. Z. 115 (1970), 117-139, MR 41 \#8401.

11. - Finitely-generated modules of finite injective dimension over certain CohenMacaulay rings, Proc. London Math. Soc. 25 (1972), 303-328.

Department of Mathematics, Københavns Universitet, Copenhagen, Denmark 